East African Medical Journal Vol. 86 (Supplement) December 2009

IMMUNOPHENOTYPING OF ACUTE LEUKAEMIAS BY FLOW CYTOMETRY: A REVIEW

R. Pamnani, MBBS, DCP (Clin. Path.), MD (Path), Lecturer, Haematology and Blood Transfusion Unit, Department of Human Pathology, College of Health Sciences, University of Nairobi, P. O Box 19676-00202, Nairobi, Kenya

\title{
IMMUNOPHENOTYPING OF ACUTE LEUKAEMIAS BY FLOW CYTOMETRY: A REVIEW
}

\author{
R. PAMNANI
}

\begin{abstract}
Objective: To provide an overview of the utility of flow cytometry for phenotyping of acute leukaemias and selection of monoclonal antibodies.

Data sources: The literature review was obtained through internet, journals and chapters in the relevant books.

Data selection: Relevant articles and chapters on immunophenotyping of acute leukaemias were selected from respected international journals and books in the field of haematology and were reviewed.

Data extraction and synthesis: Complete articles relevant to the topic were selected and reviewed and the necessary information extracted for this review.

Conclusions: Flow cytometry has been used extensively in recent years to characterise haemopoeitic malignancies and done routinely in the developed world. This technique has greatly improved the diagnosis and classification of haemopoeitic malignancies and has been recommended by World Health Organisation classification (WHO) of tumours of haemopoeitic and lymphoid tissue. Application of flow cytometry for the diagnosis of leukaemias has been recently introduced in Kenya and is currently being undertaken in research using limited but appropriate panels of monoclonal antibodies. It is hoped that findings of this research will inform the use of flow cytometry as an ancillary diagnostic technique in our resource-constrained set up.
\end{abstract}

\section{INTRODUCTION}

Flow cytometry which is a method of detecting membrane, cytoplasmic or nuclear antigens in cells produces rapid measurements of physical characteristics of cells suspended in a moving fluid stream. As many as 10,000 cells can be analysed per second. Currently in most of the developing countries, characterisation and classification of leukaemias is done by morphological and cytochemical analysis of cells in peripheral blood and bone marrow. With advances in medicine, flow cytometry has become an essential aid for accurate diagnosis, subclassification and prognostication of leukaemias and lymphomas. It is more sensitive and less subjective than morphology in analysing leukaemic cases. The World Health Organisation (WHO) classification of tumours of haemopoeitic and lymphoid tissue relies not only on morphology of the tumours but also on immunophenotyping, cytogenetics and molecular genetics for as an accurate diagnosis (1).

\section{FLOW CYTOMETRY}

The concept of flow cytometry has been there for more than 50 years and cell counters have been used for 25 years. The emergence of fluorescence detectors in the new generation of cytometers greatly improved their utility. Flow cytometry is efficient, sensitive and accurate giving more reproducible results than manual techniques. Multiple specimens can be analysed with a panel of 3-10. Testing is rapid and up to 10,000 cells can be analysed in one second.

Flow cytometry is highly sensitive making it possible to detect very small numbers of neoplastic cells. This is especially useful in detecting small numbers of leakaemic cell in the bone marrow, as a way of assessing the minimal residual disease after treatment of leukaemias.

The major limitations of flow cytometry are the high costs of instruments and reagents, specialised skills and experience required to perform the tests and sometimes difficulties of correlating the morphology 
with the markers obtained in flow cytometry.However, with the recent increase in specific monoclonal antibodies and fluorochromes, and the emergence of four to six colour analysers, the specificity of flow cytometry has markedly improved.

\section{MONOCLONAL ANTIBODIES}

A wide array of monoclonal antibodies is available for flow cytometry. Diagnosis of leukaemia is made using panels of monoclonal antibodies. This panel requires careful selection as it is the most important step towards diagnosis of leukaemia. It should be large enough to diagnose and subtype most of the leukaemias but should also be limited so as to be cost effective. These monoclonal antibodies are directed against the following category of antigens:

Lineage associated antigens: The antibodies are directed against these antigens and they help in the identification of the lineage of leukaemic cells (2). Table 1 summarises the characteristics of these antibodies.

Table 1

Lineage associated antigens

\begin{tabular}{ll}
\hline B-cell & CD10, CD19, CD20, CD21, CD22, \\
& CD23, CD24, CD79, CD138, \\
& immunoglobulin \\
T-cell & CD1, CD2, CD3, CD4, CD5 \\
& CD7, CD8, CD45RA, CD45RO, \\
& TCR \\
NK cell & CD16, CD56, CD57 \\
Myeloid & CD13, CD14, CD15, CD33, \\
& CD117, anti-MPO \\
Monocytic & CD11b, CD11c, CD14, CD64 \\
Erythroid & Glycophorin A \\
Megakaryocytic CD41, CD61 & CD \\
\hline
\end{tabular}

Immature cell antigens: These include CD10, CD34, CD117, Tdt. The presence of these markers usually indicates a haematological neoplasm.

Pan-leucocyte antigen: CD45 is the pan leucocyte marker. Side scatter properties in conjunction with CD45 expression is helpful in differential diagnosis of different leukaemia sub types. In the majority of acute leukaemias, there is low side scatter and moderate expression of CD45. In the last few years, CD45 has been used for resolving leucocyte populations and help in accurate gating.

\section{SELECTION OF PANEL OF MONOCLONAL ANTIBODIES}

Because of the high cost associated with monoclonal antibodies, judicious choice of a panel of monoclonals is recommended. Factors taken into consideration include the type of laboratory, workload of leukaemic samples, technical expertise available, number of antibodies used in the panel and others likeindication for immunophenotyping (initial diagnosis, sub typing, follow-up studies, and detection of minimal residual disease). The type of flow cy tometer (three or more colours) will also help determine the panel.

\section{APPROACHES TO PANEL SELECTION}

General, comprehensive panel: This approach is used in laboratories where cost is not a constraint. It normally includes 40-45 monoclonal antibodies per panel. The advantage of this approach is a faster turn around time, with less labourious and additional staining rarely required. It provides extensive information and minimal judgement is required. The main disadvantage is the wastage of reagents (3).

Two-tiered approach: In this approach a minimal screening panel gives basic initial information which is then followed by secondary / specific panel chosen according to results obtained with the primary panel. The approach is more labourious and time consuming and may not necessarily be cost effective in all situations (3).

Directed or targeted approach: It is based on the availability of other data such as morphologic and clinical information. This approach can be cost effective as one may use very few antibodies in the initial panel but can be dangerous if wrong clinical information is provided or a mistake has been madein reporting the morphology. Many a times, morphology could be subjective (3).

Indication based approach: This was the approach used by the 2006 Bethesda International Consensus Conference. This is quite different and utilises a combination of lineage-sensitive panel of monoclonal antibodies, (B-cell, T-cell, myeloid, monocytic, plasma cells) which is based upon the clinical presentation and medical indication (4).

\section{RECOMMENDATIONS}

Varying guidelines have been published by different working groups from different parts of the world for implementation of flow cytometry in leukaemias. 
None of them have arrived at a consensus on the way to select anideal panel of monoclonal antibodies required to analyse cases of acute leukaemias. It is possible to have an algorithm for chronic lymphoproliferative disorders but heterogeneity in the antigens and inconsistent antigen expression by the blasts makes it difficult to have the same in acute leukaemias. The British Committee for Standards in Haematology (BCSH) guideline by Bain et al (5) in 2002 recommended that if the case is clearly myeloid (based on presence of auer rod and / or with positive for myeloperoxidase or Sudan Black B), then there is not much gained with immunophenotyping. But all other cases like acute lymphoblastic leukaemia (ALL), acute myeloid leukaemia (AML)-MO, AMLM7, biphenotypic leukaemias should be diagnosed with flow cytometry.

The US-Canadian Consensus (3) was of the opinion that an initial core panel be used to identify the type of disease and the predominant cell lineage. This should be followed by a supplementary panel. It recommended CD2, CD5, CD7, CD10, CD13, CD14, CD19, CD33, CD34, HLA-DR and kappa/lambda to be used as the core panel.

The International Society for Advancement of Cytometry(ISAC) consensus meet in 2000 between US and European experts recommended CD3, CD7, CD10, CD14, CD19, CD33, CD34, CD45 and HLA$\mathrm{DR}$ be used as initial core panel along with at least one antibody of B-cell (CD20, CD22, CD79a, IgM), T-cell (CDla, CD2, CD4, CD5, CD8), myeloid (CDllb, CD15, CD64, CD117, anti-MPO), erythroid (CD36, CD71, glycophorin-A) and megakaryocytic (CD41, CD61) (6).

$\mathrm{BCSH}$ (2) recommended $\mathrm{CD} 2, \mathrm{CD} 3, \mathrm{CD} 10$, CD13, CD19, cCD22, CD79a, CD117, anti-MPO and $\mathrm{Tdt}$ as first line panel.

The Latin-American consensus conference (7) advised usage of CD13, CD15, CD33, CD117, anti-MPO (myeloid), CD10, CD19, CD79a, SIg, cytoplasmic kappa/lambda ( B lineage), CD2, CD3, CD7 (T lineage) and CD34, CD45, HLA-DR and Tdt be used.

In contrast, 2006BethesdaInternational Consensus recommendations include a panel of antibodies which are sensitive to identify cells of particular lineage. It is suitable for acute leukaemias and also chronic lymphoproliferative disorders for primary evaluation. Combination of these markers are used (B cell, T cell, myeloid) for a particular medical indication. This guideline uses CD5, CD10, CD19, CD20, CD45, kappa, lambda for B-cell, CD2, CD3, CD4, CD5, CD7, CD8, CD45, CD56 for T/ NK cell, CD7, CD1lb, CD13, CD14,
CD15, CD16, CD34, CD45, CD56, CD117, HLA-DR for myelomonocytic cells and CD19, CD38, CD45, CD56 for plasma cells. Based on the results of this primary panel, a more extensive lineage-specific panel of antibodies is used (4).

Another difficult area of diagnosis is acute biphenotypic leukaemia which has been described in detail by European group for the immunological characterisations of leukaemias (EGIL) (8). It has also been discussed by Campana and Behm (9). There are many limitations of all these recommendations.

After carefully considering all these recommendations, we have made a panel of antibodies to evaluate acute leukaemias. This panel is wide and presently useful for research purpose. The information gathered from this research will help us select appropriate antibodies for diagnostic service which will be suitable for our setting.

\section{CONCLUSION}

Over the past twenty five years, immunophenotyping has become an essential tool for characterisation of acute leukaemias. Flow cytometry is one of the immunophenotyping techniques. Morphology along with cy tochemistry may not give complete picture in all the cases and also could be subjective. They have to be complemented with flow cytometry for precise diagnosis. It is also important to remember that immunophenotypingisnotasubstitutetomorphology which still remains the gold standard for all leukaemic cases. Every laboratory has to make their own panel of antibodies which suits them. This panel should have adequate number of antibodies so that it has high sensitivity.

In Kenya, taking into consideration various recommendations and experiences, a panel of suitable monoclonal antibodies to evaluate acute leukemias has been arrived at. This panel is wide and is currently being used in flowcytometry for research of acute leukaemias. The information gathered from ongoing research will help us to select an appropriate antibodies panel to serve as an ancillary test in the diagnosis of acute leukaemias within our resource constrained setting.

\section{ACKNOWLEDGEMENTS}

To the University of Nairobi, and the American Society of Hematology (ASH), which enabled me to undertake training in flow cytometry of leukaemias and lymphomas at the M.D.Anderson Cancer Center, Houston, Texas, USA. 


\section{REFERENCES}

1. Swordlow, S.H., Campo, E., Jaffe, E.S., et al. WHO classification of tumors of haemopoeitic and lymphoid tissues. IARC. 2008.

2. Knowles, D.M. Immunophenotypic markers useful in the diagnosis and classification of hemotopoeitic neoplasms. In: Knowles D.M, ed. Neoplastic Hematopathology. 2nd ed. Philadelphia: Lippincott Williams \& Wilkins; 2001: 93-226.

3. Stewart, C.C., Behm, F.G., Carey, J.L., et al. USCanadian consensus recommendations on the immunophenotyping analysis of haematologic neoplasia by flow cytometry: Selection of antibody combinations. Cytometry. 1997; 30: 231-235.

4. Wood, B.L., Arroz, M., Barnett, D., et al. 2006 Bethesda international consensus recommendations on the immunophenotypic analysis of hematolymphoid neoplasia by flow cytometry: Optimal reagents and reporting for the flow cytometric diagnosis of hematopoeitic neoplasia. Cytometry. 2007; 72: S14-S22.

5. Bain, B.J., Barnett, D., Linch, D., Matutes, E. and Reilly, J.T. General haematology task force of the British Committee for Standards in Haematology (BCSH),
British Society of Haematology. Revised guidelines on immunophenotyping in acute leukaemia and chronic lymphoproliferative disorders. Clin. Lab. Hematol. 2002; 24:1-13.

6. Braylan, R.C., Orfao, A., Borowitz, M.J. and Davis, B.H. Optimal number of reagents required to evaluate hematolymphoid neoplasias: Results of an international consensus meeting. Cytometry. 2001;46: 23-27.

7. Ruiz-Arguelles, A., Rivadeneyra-Espinoza, L., Duque, R.E. and Orfao, A. Report on the second Latin American consensus conference for flow cytometric immunophenotyping of hematological malignancies. Cytometry. 2005; 70: 39-44.

8. Bene, M.C., Castoldi, G., Knapp, W., et al. Proposals for the immunological classification of acute leukaemias; European Group for the Immunological characterization of Leukaemias (EGIL). Leukemia. 1995; 9:1783-1786.

9. Campana, D. and Behm, F.G. Immunophenotyping of leukaemia. J. Immunol. Met. 2000; 243: 59-75. 